\title{
Rationale, Design, and Methods of the Study of Comparison of Canagliflozin vs. Teneligliptin Against Basic Metabolic Risks in Patients with Type 2 Diabetes Mellitus (CANTABILE study): Protocol for a Randomized, Parallel-Group Comparison Trial
}

\author{
Cheol Son (D) - Masato Kasahara - Tomohiro Tanaka • Noriko Satoh-Asahara • \\ Toru Kusakabe $\cdot$ Kunihiro Nishimura · Yoshihiro Miyamoto • \\ Shu Kasama · Kiminori Hosoda
}

Received: September 19, 2019 / Published online: November 1, 2019

(c) The Author(s) 2019

\section{ABSTRACT}

Background: Dipeptidyl peptidase-4 (DPP-4) inhibitors and sodium-glucose co-transporter 2 (SGLT2) inhibitors are widely used antidiabetic drugs. However, to date, no studies have directly compared the effects of these two drugs on the components of the metabolic syndrome in patients with type 2 diabetes mellitus (T2DM).

Objectives: The Comparison of Canagliflozin vs. Teneligliptin against Basic Metabolic Risks in

Cheol Son, Masato Kasahara, and Tomohiro Tanaka have contributed equally to this research.

Enhanced Digital Features To view enhanced digital features for this article go to https://doi.org/10.6084/ m9.figshare.9976166.

C. Son $(\bowtie) \cdot$ K. Hosoda

Division of Diabetes and Lipid Metabolism,

National Cerebral and Cardiovascular Center, Suita,

Osaka, Japan

e-mail: son@ncvc.go.jp

M. Kasahara · S. Kasama

Institute for Clinical and Translational Science,

Nara Medical University Hospital, Kashihara, Nara, Japan

T. Tanaka

Department of Gastroenterology and Metabolism, Nagoya City University Graduate School of Medical Sciences, Nagoya, Aichi, Japan
Patients with T2DM (CANTABILE) study aims to examine whether the DPP-4 inhibitor (teneligliptin) or the SGLT2 inhibitor (canagliflozin) is the more effective drug for reducing metabolic risk factors as a composite in Japanese patients with T2DM.

Methods: The CANTABILE study is a prospective, multicenter, open-label, randomized, parallel-group comparison study. A total of 200 patients with T2DM treated with metformin alone or without glucose-lowering agents will be enrolled if they have one or more of the metabolic risk factors, such as obesity, borderline high blood pressure, and dyslipidemia. They will then will be randomized into the Teneligliptin group or the Canagliflozin group and treated for 24 weeks. The primary endpoint is the composite ratio of subjects with one or more improved metabolic risk factors. The

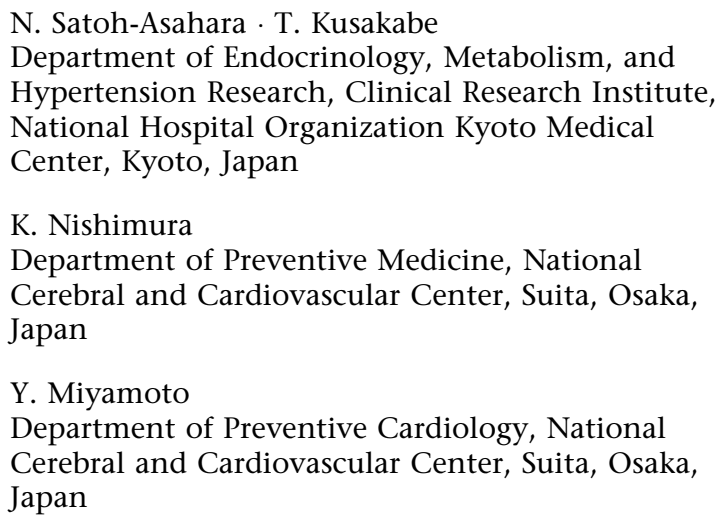


secondary endpoints are the changes in each component of the primary endpoint.

Planned outcomes: The CANTABILE study provides valuable evidence to indicate the suitability of SGLT2 inhibitors or DPP-4 inhibitors for Japanese patients with T2DM and metabolic risks.

Trial Registration Number: University Hospital Medical Information Network Clinical Trial Registry number: UMIN000030343.

Funding: Mitsubishi Tanabe Pharma Corporation.

Keywords: Canagliflozin; Metabolic syndrome; Teneligliptin; Type 2 diabetes mellitus; Risk factors

\section{Key Summary Points}

Why carry out this study?

Dipeptidyl peptidase-4 (DPP-4) inhibitors and sodium-glucose co-transporter 2 (SGLT2) inhibitors are widely-used antidiabetic drugs.

However, no studies have directly compared the effects of these two drugs on the components of the metabolic syndrome in patients with type 2 diabetes mellitus (T2DM).

The objectives of this study is to examine whether the DPP-4 inhibitor (Teneligliptin) or the SGLT2 inhibitor (Canagliflozin) is more effective for the reduction of metabolic risk factors as a composite in Japanese patients with T2DM.

\section{What will be learned from the study?}

This study is a prospective, multicenter, open-label, randomized, parallel group, comparison study.

This study will provide valuable evidence to indicate the suitability of SGLT2 inhibitors or DPP-4 inhibitors for Japanese patients with T2DM and metabolic risks.

\section{INTRODUCTION}

Type 2 diabetes mellitus (T2DM) is associated with multiple vascular complications and represents one of the major threats worldwide to life expectancy and quality of life [1]. Therefore, rigorous efforts have been made to lower blood glucose levels and prevent diabetic complications. Randomized controlled trials have consistently shown that the reduction of glycated hemoglobin (HbA1c) levels does not effectively decrease the risk for cardiovascular events, although it does prevent microvascular events [2-5].

In contrast with studies that have aimed to achieve intensive glycemic control, trials with the target of simultaneously controlling glucose, blood pressure, and lipids have shown that multifactorial interventions can reduce cardiovascular complications and mortality in patients with T2DM $[6,7]$. When these metabolic risk factors cluster in a single person, as often happens, the risk for cardiovascular events is synergistically, rather than additively, enhanced [8]. In one study, a higher prevalence of metabolic syndrome was found in Japanese patients with T2DM [9]; in addition, the Casale Monferrato Study reported a higher cardiovascular risk in patients with T2DM with at least one component of metabolic syndrome [10]. These findings indicate that it is plausible to concomitantly control metabolic risk factors during the treatment of patients with T2DM.

Recent years have witnessed the appearance of many new classes of antidiabetic drugs that have provided more options in the pharmacotherapy of diabetes mellitus. Groundbreaking prospective studies have very recently shown that sodium-glucose co-transporter 2 (SGLT2) inhibitors [11-13] and glucagon-like peptide-1 (GLP-1) agonists [14, 15] significantly reduce cardiovascular risk. Although it remains uncertain whether positive results in these studies reflect the multifactorial effect of these drugs, diabetes treatment has entered into a new era, where the choice of drug is more important than the actual lowering of blood glucose levels. However, participants enrolled in these abovementioned prospective studies were either those 
with cardiovascular disease already present $[11,12]$ or those at extreme risk for developing cardiovascular disease $[12,13]$.

Dipeptidyl peptidase-4 (DPP-4) inhibitors are incretin-related drugs that exert glucose-lowering effects with a low risk of hypoglycemia [16]. A meta-analysis of 55 clinical trials showed that DPP-4 inhibitors exhibit a stronger glucoselowering effect in Asians than in other ethnic groups [17]. Accordingly, since their launch in 2009 in Japan, DPP-4 inhibitors have been established as one of the most frequently used antidiabetic drugs in Japan [18]. Although some studies have shown the beneficial effects of DPP-4 inhibitors in the prevention of the progression of atherosclerotic lesions [19, 20], DPP4 inhibitors are generally thought to have neutral effects on cardiovascular events [18]. However, analysis of the overall effects of DPP-4 inhibitors on components of the metabolic syndrome that constitute the metabolic risk complex in diabetic patients has not yet been undertaken.

SGLT2 inhibitors are a relatively new class of antidiabetic drug that lead to a continuous excretion of glucose in the urine [21], which results in body weight loss and reduced blood glucose levels. Recent large-scale clinical trials have reported that SGLT2 inhibitors decrease cardiovascular events in patients with T2DM with high cardiovascular risk [11, 12], but the effects of SGLT2 inhibitors on early-phase patients with T2DM accompanied with multiple metabolic risks have not been clarified.

Based on this background, the aim of the CANTABILE (Comparison of Canagliflozin vs. Teneligliptin against Basic Metabolic Risks in Patients with T2DM) study is to examine whether the DPP-4 inhibitor (teneligliptin) or the SGLT2 inhibitor (canagliflozin) is the more effective drug for reducing metabolic risk components as a composite in Japanese patients with T2DM. The study is so designed that its results are expected to address the as-yet unanswered question of how these two drugs can properly be used in relatively low-risk, earlyphase patients who are drug naive or receive metformin monotherapy.

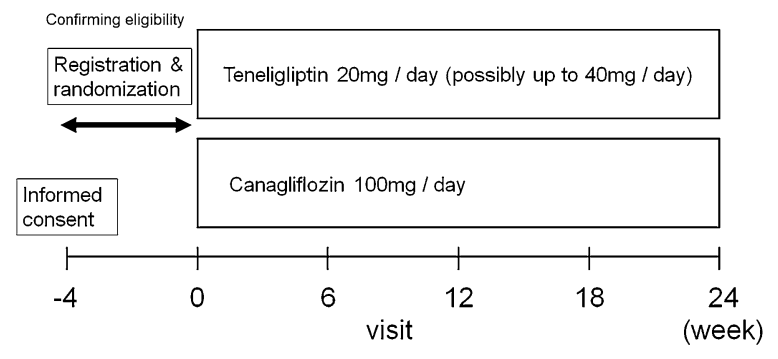

Fig. 1 Study design. A total of 200 patients with type 2 diabetes mellitus (100 patients/group) will participate in this study. After the confirmation of eligibility by a central managing modality, the enrolled patients will be randomly assigned to canagliflozin or teneligliptin therapy. In the canagliflozin group, $100 \mathrm{mg}$ canagliflozin will be orally administered once per day. In the teneligliptin group, $20 \mathrm{mg}$ Teneligliptin will be orally administered once per day. If necessary, the teneligliptin dose can be increased to up to $40 \mathrm{mg}$ per day

\section{METHODS}

\section{Study Design}

This is a prospective, multicenter, open-label, randomized, parallel-group comparison study. It is an exploratory study to compare the influence of canagliflozin/teneligliptin treatment on the rate of improvement in metabolic risk factors after 24 weeks of treatment (Fig. 1). We define the metabolic risks as obesity, borderlinehigh blood pressure, and dyslipidemia. The cutoff values of each risk factor are to be determined according to the Japanese criteria for the diagnosis of metabolic syndrome [22]. The qualifying patients are to be randomly assigned to canagliflozin or teneligliptin therapy (see section "Treatments"). A glycemic (HbA1c) goal is set as $<53 \mathrm{mmol} / \mathrm{mol}$ (7.0\%). In principle, diet, exercise therapy, and cardiovascular medications ongoing prior to the study will continue without modification. The changes in body weight, blood pressure, and triglyceride (TG) and high-density lipoprotein-cholesterol (HDL-C) levels will be evaluated after 6, 12, 18, and 24 weeks of treatment.

This study will be conducted at 38 institutions in Japan in compliance with both the articles of the Declaration of Helsinki (revised in 
Table 1 Primary and secondary endpoints of the CANTABILE study

\begin{tabular}{|c|c|}
\hline Primary endpoint & Secondary endpoints \\
\hline $\begin{array}{l}\text { Composite ratio of subjects with improvement in } \geq 1 \text { of the } \\
\text { following metabolic risk factors: } \\
\text { 1. BMI } \geq 25 \mathrm{~kg} / \mathrm{m}^{2} \text { at week } 0 \text { of treatment and } \geq 3 \% \\
\text { weight loss after } 24 \text { weeks of treatment } \\
\text { 2. SBP } \geq 130 \mathrm{mmHg} \text { or DBP } \geq 85 \mathrm{mmHg} \text { at week } 0 \text {, and } \\
\mathrm{SBP}<130 \mathrm{mmHg} \text { and } \mathrm{DBP}<85 \mathrm{mmHg} \text { after } \\
24 \text { weeks of treatment } \\
\text { 3. Fasting TG } \geq 1.69 \mathrm{mmol} / \mathrm{L} \text { or } \mathrm{HDL}-\mathrm{C}<1.03 \mathrm{mmol} / \mathrm{L} \\
\text { at week } 0 \text {, and fasting } \mathrm{TG} \text { of }<1.69 \mathrm{mmol} / \mathrm{L} \text { and } \mathrm{HDL} \text { - } \\
\mathrm{C} \geq 1.03 \mathrm{mmol} / \mathrm{L} \text { after } 24 \text { weeks of treatment }\end{array}$ & $\begin{array}{l}\text { 1. The ratio of the number of participants who meet the } \\
\text { primary criteria consisting of } 1,2 \text {, and } 3 \text { listed in the } \\
\text { primary endpoint } \\
\text { 2. HbAlc change from baseline } \\
\text { 3. Fasting blood glucose change from baseline } \\
\text { 4. Achievement ratio of } \mathrm{HbAlc}<42 \mathrm{mmol} / \mathrm{mol}(6.0 \%) \text {, } \\
\text { and achievement ratio of } \mathrm{HbAlc}<53 \mathrm{mmol} / \mathrm{mol}(7.0 \%) \\
\text { 4. Achievement ratio of } \mathrm{HbAlc}<42 \mathrm{mmol} / \mathrm{mol}(6.0 \%) \text {, } \\
\text { and achievement ratio of } \mathrm{HbA} 1 \mathrm{c}<53 \mathrm{mmol} / \mathrm{mol}(7.0 \%) \\
\text { 5. Achievement ratio of } \geq 3 \% \text { weight loss, and achievement } \\
\text { ratio of } \geq 5 \% \text { weight } 1 \text { oss } \\
\text { 6. Changes from baseline in waist circumference, BMI, and } \\
\text { body weight } \\
\text { 7. Changes from baseline in HDL-C and fasting TG } \\
\text { 8. Changes from baseline in blood pressure (SBP and DBP) }\end{array}$ \\
\hline
\end{tabular}

$B M I$ Body mass index, $D B P$ diastolic blood pressure, $H b A l c$ glycated hemoglobin, $H D L-C$ high-density lipoprotein cholesterol, $S B P$ systolic blood pressure, $T G$ triglyceride

October 2013) and the Ethical Guidelines for Medical and Health Research Involving Human Subjects established by the Ministry of Health, Labor, and Welfare in Japan. Independent ethics committees at each institute approved the study protocol. The registration period is from 1 October 2017 to 31 March 2019, and the research period is from 1 October 2017 to 30 September 2020. In accordance with the law for clinical research in Japan in April 2018, the protocol of this study underwent reexamination and was approved by the Nara Medical University Certified Review Board (approval number: nara0002).

\section{Planned Outcomes}

\section{Primary Endpoint}

The primary endpoint is the composite ratio of subjects with improvement in one or more of the following metabolic risk factors (Table 1):
- $\quad$ BMI $\geq 25 \mathrm{~kg} / \mathrm{m}^{2}$ at week 0 of treatment and $\geq 3 \%$ weight loss after 24 weeks of treatment.

- $\quad$ Systolic blood pressure $(\mathrm{SBP}) \geq 130 \mathrm{mmHg}$ or diastolic blood pressure (DBP) $\geq 85 \mathrm{mmHg}$ at week 0 , and SBP $<130 \mathrm{mmHg}$ and DBP $<85 \mathrm{mmHg}$ after 24 weeks of treatment.

- Fasting TG $\geq 1.69 \mathrm{mmol} / \mathrm{L}$ or HDL$\mathrm{C}<1.03 \mathrm{mmol} / \mathrm{L}$ at week 0 , and fasting TG of $<1.69 \mathrm{mmol} / \mathrm{L}$ and HDL-C $\geq 1.03 \mathrm{mmol} /$ $\mathrm{L}$ after 24 weeks of treatment.

\section{Secondary Endpoints}

The secondary endpoints are listed in Table 1.

- Safety endpoints: Data on the clinical investigation results, vital sign measurements, and adverse events (AEs) will be collected at each visit. The severity and causal 
Table 2 Inclusion and exclusion criteria of the CANTABILE study

Inclusion criteria
1. Patients providing written informed consent to participate
in the study
2. Patients aged $\geq 20$ and $<85$ years (independent of sex)
3. HbAlc $\geq 53 \mathrm{mmol} / \mathrm{mol}(7.0 \%)$ and $<86 \mathrm{mmol} / \mathrm{mol}$
$(10.0 \%)$

4. Patients who have at least one of following metabolic risk factors:

a) $\mathrm{BMI} \geq 25 \mathrm{~kg} / \mathrm{m}^{2}$

b) $\mathrm{SBP} \geq 130 \mathrm{mmHg}$ or $\mathrm{DBP} \geq 85 \mathrm{mmHg}$

c) $\mathrm{TG} \geq 1.69 \mathrm{mmol} / \mathrm{L}$ or HDL-C $<1.03 \mathrm{mmol} / \mathrm{L}$

5. Patients who have not changed the antidiabetic therapy as specified for at least 8 weeks before the date of informed consent:

a) Diet therapy and/or exercise therapy with metformin monotherapy

b) Diet therapy and/or exercise therapy with no antidiabetic medications

\section{Exclusion criteria}

1. Type 1 diabetes mellitus

2. $\mathrm{BMI}<22 \mathrm{~kg} / \mathrm{m}^{2}$

3. Hypersensitivity to contents of teneligliptin or canagliflozin

4. Require insulin therapy for blood glucose management

5. Congestive heart failure (NYHA III or IV)

6. Women who are pregnant, breast-feeding, or may be pregnant

7. Diagnosed or suspected malignant tumors

8. Taking unallowed medications or undertaking unallowed therapy as defined in the study protocol during the preceding 8 weeks

Contraindicated concomitant medications: insulin, sulfonylurea, glinide, alpha-glucosidase inhibitor, biguanide (exception of metformin), thiazolidine, DPP-4 inhibitor, SGLT2 inhibitor, GLP-1 receptor agonist, combination products including these active components, and corticosteroid for systemic effects.

Contraindicated concomitant therapies: bariatric surgery

9. Changes in dosage of concomitantly administered drugs or therapy content during the preceding 8 weeks Limited concomitant medications: metformin hydrochloride, antihypertensives, diuretics, medicines for dyslipidemia, and medicines for obesity, including the Chinese medicine "bouhuutsuushousan"

Limited concomitant therapies: diet therapy, exercise therapy

10. Patients who were judged by the investigator to be inappropriate for the study

DDP-4 Dipeptidyl peptidase 4, GLP-1 glucagon-like peptide-1, NYHA New York Heart Association functional classification, SGLT2 sodium-glucose transport protein 2 
Table 3 Rules for concomitant medications

\section{Concomitant medications}

Contraindicated concomitant medications ${ }^{a}$

Continued concomitant medications

1. Insulin

2. Sulfonylurea

3. Glinide

4. Alpha-glucosidase inhibitor

5. Biguanide (exception of metformin)

6. Thiazolidine

7. DPP-4 inhibitor (with the exception of TENELIA ${ }^{\circledR}$ tablets $20 \mathrm{mg}$ for the Teneligliptin group during the study)

8. SGLT2 inhibitor (with the exception of CANAGLU ${ }^{\circledR}$ tablets $100 \mathrm{mg}$ for the Canagliflozin group during the study)

9. GLP-1 receptor agonist

10. Combination products that include active components (2) to (9) mentioned above

11. Corticosteroids

12. Bariatric surgery (gastric bypass surgery, gastric banding surgery, and intra-gastric balloon placement)

a Contraindicated from at least 8 weeks before the date of informed consent until week 24 of treatment

b Continued without modification from at least 8 weeks before the date of informed consent until week 24 of treatment

relationship of all AEs to the study drug and outcomes of all AEs will be assessed.

- Other confirmation items: The results of the Framingham Risk Score and of the brief-type self-administered diet history questionnaire (BDHQ) will be used as additional information for further discussion.

\section{Study Population}

In total, 200 patients with T2DM will participate in this study. The participants in the study must have insufficient glycemic control under diet and exercise regimens and/or treatment with or without metformin. Patients will be considered eligible for the study if they fulfil all of the inclusion criteria and none of the exclusion criteria, as defined in Table 2. The investigator will provide a sufficient explanation of the
1. Metformin hydrochloride

2. Antihypertensives

3. Diuretics

4. Medicines for dyslipidemia

5. Medicines for obesity, including the Chinese medicine "Bouhuutsuushousan" study to each participant and obtain written informed consent.

\section{Randomization}

The registration and allocation of the study participants will be performed by the central registration modality using an electronic data capture (EDC) system. After confirmation of the eligibility of the participants, the investigator will input the participant's data into the EDC system, and if the eligibility is confirmed, the participant's identification code will be assigned. Participants whose eligibility has been confirmed will be dynamically assigned to either the Teneligliptin group or the Canagliflozin group by using the following assignment factors: HbA1c on the date of consent, fasting TG, BMI, SBP, and whether or not metformin was administered. 


\section{Treatments}

In the Canagliflozin group, $100 \mathrm{mg}$ canagliflozin $\left(\right.$ CANAGLU $^{\circledR}$ tablets $100 \mathrm{mg}$; Mitsubishi Tanabe Pharma) will be orally administered in addition to the participant's ongoing diabetic treatment. In the Teneligliptin group, $20 \mathrm{mg}$ teneligliptin (TENELIA ${ }^{\circledR}$ tablets $20 \mathrm{mg}$; Mitsubishi Tanabe Pharma) will be orally administered in addition to the participant's ongoing diabetic treatment; if necessary, the teneligliptin dose can be increased to up to $40 \mathrm{mg}$ per day. The concomitant diet and exercise therapies ongoing prior to the study will continue without modification from at least 8 weeks before the date of informed consent until week 24 of treatment. The rules for concomitant medications are detailed in Table 3.

\section{Measurements}

The schedule of data collection is detailed in Table 4.

\section{Sample Size Calculation}

Based on the results of previous clinical trials on canagliflozin and teneligliptin, if the rate of primary endpoint of canagliflozin and teneligliptin achieved was $65: 40 \%$ or $70: 45 \%$, the sample size required to detect this difference using a Chi-squared test $(1-\beta=0.9, \alpha=0.05$ two-sided) is estimated to be $81-82$ cases per group. Taking into consideration a $10 \%$ dropout rate during the study, the target number of patients is set at 90-92 in each group for a total of $180-184$ patients.

\section{Data Analysis}

\section{Analysis Populations}

For the evaluation of efficacy, two analysis groups will be defined: a full analysis set (FAS) and a per protocol set (PPS), with FAS to be used for the primary analysis. The inclusion of patients into each analysis set (FAS, PPS, and safety analysis set [SAS]) will be performed under circumstances that mask the medications being studied.

- The FAS is defined as the participant population registered with informed consent and with a measured value of the factors of the primary endpoint (body weight, SBP, DBP, fasting TG, and HDL-C) at baseline and for at least one time point during the treatment period.

- The PPS is defined as the participant population excluded from the FAS for any of the following:

(a) violation of safety or human rights-related exclusion criteria, meeting criteria for study discontinuation.

(b) non-compliance with allocated drugs, violation of effectiveness-related inclusion criteria, administration of prohibited treatment, study drug compliance rate $<70 \%$, or treatment period $<18$ weeks.

- The SAS will include patients for whom safety evaluation data were collected after the start of study treatment.

\section{Statistical Methods}

The pooled patients receiving the doses of 20 and $40 \mathrm{mg}$ teneligliptin, respectively, are defined as the Teneligliptin group. The summary statistics and frequency of each category for categorical factors will be calculated for each baseline value and for body weight, SBP, DBP, TG, and HDL-C at week 24 in both groups. For the changes in body weight, SBP, DBP, TG, and HDL-C level from baseline, the least-squares mean (LS-MEAN) difference and the 95\% confidence interval $(95 \% \mathrm{CI})$ will be calculated to compare the groups, using analysis of covariance (ANCOVA), with the baseline value as a covariate. The difference in rate will also be tested. Subgroup analysis will be performed to assess the factors influencing the primary endpoint. For the continuous variables of the secondary endpoints, summary statistics will be calculated for each measurement value and the 
Table 4 Schedule of enrollment, interventions, and assessments (SPIRIT flow diagram)

\begin{tabular}{|c|c|c|c|c|c|c|c|}
\hline \multirow[b]{3}{*}{ TIMEPOINT } & \multicolumn{7}{|c|}{ STUDY PERIOD } \\
\hline & \multirow{2}{*}{$\begin{array}{c}\begin{array}{c}\text { Enrol } \\
\text { ment }\end{array} \\
-4 W\end{array}$} & \multirow{2}{*}{$\begin{array}{c}\text { Allocation } \\
0\end{array}$} & \multicolumn{4}{|c|}{ Post-allocation } & \multirow[t]{2}{*}{ Close-out } \\
\hline & & & $6 W$ & $12 W$ & $18 W$ & $24 W$ & \\
\hline \multicolumn{8}{|l|}{ ENROLMENT: } \\
\hline Eligibility screen & X & & & & & & \\
\hline Informed consent & $\mathrm{X}$ & & & & & & \\
\hline Allocation & & $\mathrm{X}$ & & & & & \\
\hline \multicolumn{8}{|l|}{ INTERVENTIONS: } \\
\hline \multicolumn{8}{|l|}{ Teneligliptin } \\
\hline \multicolumn{8}{|l|}{ Canagliflozin } \\
\hline \multirow{2}{*}{$\begin{array}{l}\text { ASSESSMENTS: } \\
\text { participant characteristics* }\end{array}$} & & & & & & & \\
\hline & $\mathrm{X}$ & & & & & & \\
\hline vital signs** & $\mathrm{X}$ & $\mathrm{X}$ & $\mathrm{X}$ & $\mathrm{X}$ & $\mathrm{X}$ & $\mathrm{X}$ & $\mathrm{X}$ \\
\hline blood test*** & $X$ & $X$ & $X$ & $X$ & $X$ & X & $X$ \\
\hline urine test**** & & $\mathrm{X}$ & $\mathrm{X}$ & $\mathrm{X}$ & $X$ & $\mathrm{X}$ & $\mathrm{X}$ \\
\hline \multirow{4}{*}{$\begin{array}{l}\text { diet / exercise } \\
\text { therapy }\end{array}$} & & $\mathrm{X}$ & & & & & \\
\hline & & $\mathrm{X}$ & $\mathrm{X}$ & $\mathrm{X}$ & $\mathrm{X}$ & $\mathrm{X}$ & $\mathrm{X}$ \\
\hline & & & $\mathrm{X}$ & $\mathrm{X}$ & $\mathrm{X}$ & $\mathrm{X}$ & $\mathrm{X}$ \\
\hline & & $\mathrm{X}$ & & & & $\mathrm{X}$ & $\mathrm{X}$ \\
\hline
\end{tabular}

*Participant characteristics include as follows; sex, age, duration of diabetes mellitus, concomitant medications ${ }^{* *}$ Vital signs include as follows; blood pressure, pulse rate, height, body weight, BMI, waist circumference

${ }^{* * *}$ Blood test include the following items;HbAlc, fasting triglyceride, HDL-C, fasting blood glucose, white blood cell count, red blood cell count, hemoglobin content, hematocrit, $\mathrm{MCV}, \mathrm{MCH}, \mathrm{MCHC}$, platelet count, total protein, albumin, blood urea nitrogen, serum creatinine, serum uric acid, eGFR, AST, ALT, ALP, $\gamma$-GTP, TC, LDL-C, CK, Na, K, Cl, Ca, P, fasting insulin, blood ketone body fractions

${ }^{* * * *}$ Urine test includes the following items; qualitative test (protein, glucose, urobilinogen, bilirubin, ketone body, occult blood), specific gravity, $\mathrm{pH}$ 
changes from the baseline. The value of HbA1c is placed in a contingency table by the cutoff value $(42 \mathrm{mmol} / \mathrm{mol} \quad[6.0 \%], \quad 53 \mathrm{mmol} / \mathrm{mol}$ [7.0\%]), and each frequency, percentage, and 95\% CI will be calculated. For the intergroup comparison of the continuous variables, the difference in the LS-MEAN and the 95\% CI will be estimated by using ANCOVA with the baseline value as a covariate. Appropriate data conversion will be applied to data for which normal distribution is not observed. The significance level for statistical tests shall be 0.05 on both sides.

\section{STRENGTHS AND LIMITATIONS}

In the study described here, we will compare the effect of canagliflozin and teneligliptin on the improvement in metabolic risk factors after 24 weeks of treatment. The primary endpoint is the rate of improvement in one or more of the following three metabolic factors: (1) BMI $>25$; (2) $\mathrm{SBP} \geq 130 \mathrm{mmHg}$ or $\mathrm{DBP} \geq 85 \mathrm{mmHg}$; (3) $\mathrm{TG} \geq 1.69 \mathrm{mmol} / \mathrm{L}$ or HDL-C $<1.03 \mathrm{mmol} / \mathrm{L}$.

Previous studies that investigated whether better glycemic control would reduce the risk of the cardiovascular complications of diabetes mellitus, such as myocardial infarction, have reported unfavorable results [23]. These clinical trials, including the United Kingdom Prospective Diabetes Study (UKPDS) [2], the Action to Control Cardiovascular Risk in Diabetes (ACCORD) study [3], the Veterans Affairs Diabetes Trial (VADT) [4], and the Action in Diabetes and Vascular Disease: Preterax and Diamicron Modified Release Controlled Evaluation (ADVANCE) study [5], have found only limited, if any, correlation between glycemic control and diabetic cardiovascular events. The ACCORD study [3] even showed that the use of intensive therapy to achieve normal glycated hemoglobin levels increased mortality and did not significantly reduce major cardiovascular events. In contrast, the trials with multifactorial interventions, such as the STENO-2 study [6], found reduced cardiovascular complications and mortality in patients with T2DM, and the J-DOIT3 study [7] identified similar tendencies in the Japanese population. These data indicate that the control of comorbid risk factors is recommended in addition to glucose-lowering therapy for the treatment of diabetes mellitus.

SGLT2 inhibitors are a unique class of antidiabetic drugs that mainly act on renal proximal tubule cells and inhibit the SGLT2-dependent reabsorption of glucose [21]. Recent prospective large clinical trials have demonstrated the beneficial effects of this class of medications on cardiovascular events as a composite outcome [11-13]. However, these trials mainly enrolled patients with T2DM who were taking multiple medications. In the EMPA-REG OUTCOME study [11], 73, 43, and $49 \%$ of participants received metformin, sulfonylurea, and insulin, respectively; in the CANVAS study [12], the proportion of patients receiving each drug was 77,44 , and 50\%; and in the DECLARE-TIMI 58 study, 82, 43, and $41 \%$ of patients received each drug, respectively [13]. Thus, it is still unclear which effects SGLT2 inhibitors have on patients with T2DM who are either drug naive or only taking metformin in terms of primary prevention in the earlier phase of disease.

DPP-4 is a circulating enzyme that plays a role in the degradation of GLP-1 and glucose-dependent insulinotropic peptide (GIP). DPP-4 inhibition, which enhances the concentration of GLP1 and GIP, leads to the amplification of hyperglycemia-induced insulin secretion and eventually lowers blood glucose levels [16]. As neither GLP-1 nor GIP exerts its effect in hypoglycemia, DPP-4 inhibitors are generally accepted as "safe" drugs, with a low risk of hypoglycemia, especially when used as monotherapy [24].

In a retrospective registry study in Taiwan, patients with T2DM prescribed DPP-4 inhibitors were found to have a reduced risk of coronary heart disease, cerebral infarction, and overall mortality compared with those not treated with DPP-4 inhibitors [25]. It is generally accepted that DPP-4 inhibitors have a neutral effect on body weight, but they may also have beneficial effects on some metabolic disorders, such as hypertriglyceridemia [26] and hepatic steatosis [27], and they may exert anti-inflammatory effects in macrophages [28]. However, the analysis of the effects of DPP-4 inhibitors on the metabolic risks as a whole has not yet been undertaken. Considering the observed beneficial effects of DPP-4 
inhibitors on the progression of atherosclerosis of patients without cardiovascular diseases [19], it is tempting to speculate that the effect of DPP-4 inhibitors on cardiovascular risk may be more prominent in the earlier phase of vascular diseases. Prospective studies examining late-stage endpoints, such as heart failure, myocardial infarction, stroke, or death, may not be suitable for the observation of drug effects on the earlier, more metabolic stages of the cardiovascular diseases. In this context, it is of interest to examine the potential effects of DPP-4 inhibitors on metabolic risk reduction, an earlier phase endpoint in drug-naïve patients or patients receiving metformin monotherapy in this study.

There are several criteria for obesity, hypertension, and dyslipidemia. For elevated blood pressure and dyslipidemia, we used the cutoff values for the Japanese criteria for the diagnosis of the metabolic syndrome [22]. For obesity, we used the Japanese cutoff value for obesity, which differs from that of the World Health Organization (WHO) [29] but is in accordance with the suggested criteria for Asian population by the WHO expert consultant panel [30].

Although this study has several limitations, including a short observation period, a small number of patients, and an open-label modality, it will provide valuable evidence by which to identify the suitability of SGLT2 inhibitors or DPP-4 inhibitors for Japanese patients with T2DM and components of the metabolic syndrome and potentially also for the patients of East Asian origin.

\section{ACKNOWLEDGEMENTS}

The authors would like to thank all of the people who participated in this study.

Funding. Mitsubishi Tanabe Pharma Corporation funded this study and supported the protocol preparation. Mitsubishi Tanabe Pharma Corporation also funded the Rapid Service Fee. All authors had full access to all of the data in this study and take complete responsibility for the integrity of the data and accuracy of the data analysis.
Authorship. All named authors meet the International Committee of Medical Journal Editors (ICMJE) criteria for authorship for this article, take responsibility for the integrity of the work as a whole, and have given their approval for this version to be published.

Authorship Contributions. All authors are involved in the planning and execution of the CANTABILE study. CS, MK, and TT are mainly responsible for drafting the manuscript with help of SK. NSA, TK, and YM especially contributed to the planning the study. NK is responsible for statistical design and analysis. SK and $\mathrm{KH}$ critically reviewed the manuscript. MK and $\mathrm{KH}$ supervised the study.

Disclosures. Cheol Son received grants from MSD Contribution, Sanofi K.K., Eli Lilly Japan K.K., Novartis Pharma K.K, and Takeda Research Support. Masato Kasahara received compensation for the advisor of the medical consulting firm, Reason Why Inc.; payments for lectures from Fuji Yakuhin, Pfiezer, Daiichi Sankyo Co., Teijin, Fuji film, Baxter, and Otsuka Pharmaceutical; and grants from Mitsubishi Tanabe Pharma Corporation, Daiichi Sankyo Co., and Fuji yakuhin. Tomohiro Tanaka holds stocks of Sumitomo Dainippon Pharma Co., Astellas Pharma Inc.; received payment for lectures from Taisho Toyama Pharmaceutical Co., Daiichi Sankyo Co., Mitsubishi Tanabe Pharma Corp., AstraZeneca, EA Pharma Co., Kissei Pharmaceutical Co., Novartis Pharma, Takeda Pharmaceutical Co., Boehringer Ingelheim Japan, Kowa Pharmaceutical Co., MSD K.K., Astellas Pharma Inc., Ono Pharmaceutical Co., Amgen Astellas BioPharma K.K., Medtronic Co., Novo Nordisk Pharma, Sanwa Kagaku Kenkyusho, Co., Eli Lilly Japan K.K., the Japanese Society of Internal Medicine, and Aichi Prefecture Insurance Medical Association; and also honoraria for a manuscript from Taisho Toyama Pharmaceutical Co., Nippon Rinsho Sha Co., Yodosha Co., Hokuryukan \& NEW SCIENCE Co., SHINDAN TO CHIRYO SHA, Inc., and Kyoto University Press. Tomohiro Tanaka also received grants from Mitsubishi Tanabe Pharma Corp., Takeda Pharmaceutical Co., Kamitsure Laboratory Co., Smoking Research Foundation, Japan Society 
for the Promotion of Science, Kowa Pharmaceutical Co., Eli Lilly Japan K.K., MSD K.K., Ono Pharmaceutical Co., Novo Nordisk Pharma, Taisho Toyama Pharmaceutical Co., Novartis Pharma, and Sanofi K.K. Toru Kusakabe received payment for lectures from Kowa Pharmaceutical Co.; and grants from Grants-in-Aid for Scientific research, Japan Agency for Medical Research and Development, Smoking Research Foundation, Daiwa Securities Health Foundation, Mitsubishi Tanabe Pharma Corp., Eli Lilly Japan Co., TANITA Co., Novartis Pharma Co., Cosmic Co., Teijin Pharma, and Daiichi Sankyo Co. Kunihiro Nishimura received grants from Philips Japan Co., Terumo Co., and Daiichi Sankyo Co. Shu Kasama received grants from Mitsubishi Tanabe Pharma Corporation. Kiminori Hosoda received grants from MSD Contribution, Sanofi K.K., Eli Lilly Japan K.K., Novartis Pharma K.K., Takeda Research Support, Japan Cardiovascular Research Foundation, and Japan Diabetes Foundation. Noriko Satoh-Asahara and Yoshihiro Miyamoto have nothing to dsiclose.

Compliance with Ethics Guidelines. In accordance with the law for clinical research in Japan, the protocol of this study was approved by the Nara Medical University Certified Review Board (Approval Number; nara0002). This study is conducted in compliance with the articles of the Declaration of Helsinki (revised in October 2013) and in accordance with the Ethical Guidelines for Medical and Health Research Involving Human Subjects established by the Ministry of Health, Labor, and Welfare in Japan. This study is also conducted in accordance with the law for clinical research in Japan (the Clinical Trials Act). In accordance with the Japanese Clinical Trials Act, this study is also registered in the Japan Registry of Clinical Trials (jRCTs051180112).

Open Access. This article is distributed under the terms of the Creative Commons Attribution-NonCommercial 4.0 International License (http://creativecommons.org/licenses/ by-nc/4.0/), which permits any noncommercial use, distribution, and reproduction in any medium, provided you give appropriate credit to the original author(s) and the source, provide a link to the Creative Commons license, and indicate if changes were made.

\section{REFERENCES}

1. King RJ, Grant PJ. Diabetes and cardiovascular disease: pathophysiology of a life-threatening epidemic. Herz. 2016;41(3):184-92.

2. [No authors listed]. Intensive blood-glucose control with sulphonylureas or insulin compared with conventional treatment and risk of complications in patients with type 2 diabetes (UKPDS 33). UK Prospective Diabetes Study (UKPDS) Group. Lancet. 1998;352(9131):837-53.

3. Action to Control Cardiovascular Risk in Diabetes Study Group, Gerstein HC, Miller ME, et al. Effects of intensive glucose lowering in type 2 diabetes. N Engl J Med. 2008;358(24):2545-59.

4. Duckworth W, Abraira C, Moritz T, et al. Glucose control and vascular complications in veterans with type 2 diabetes. N Engl J Med. 2009;360(2):129-39.

5. ADVANCE Collaborative Group, Patel A, MacMahon $\mathrm{S}$, et al. Intensive blood glucose control and vascular outcomes in patients with type 2 diabetes. N Engl J Med. 2008;358(24):2560-72.

6. Gaede P, Valentine WJ, Palmer AJ, et al. Cost-effectiveness of intensified versus conventional multifactorial intervention in type 2 diabetes: results and projections from the Steno- 2 study. Diabetes Care. 2008;31(8):1510-5.

7. Ueki K, Sasako T, Okazaki Y, et al. Effect of an intensified multifactorial intervention on cardiovascular outcomes and mortality in type 2 diabetes (J-DOIT3): an open-label, randomised controlled trial. Lancet Diabetes Endocrinol. 2017;5(12): 951-64.

8. Matsuzawa Y, Funahashi T, Nakamura T. The concept of metabolic syndrome: contribution of visceral fat accumulation and its molecular mechanism. J Atheroscler Thromb. 2011;18(8):629_ 39.

9. Sone $\mathrm{H}$, Mizuno S, Fujii $\mathrm{H}$, et al. Is the diagnosis of metabolic syndrome useful for predicting cardiovascular disease in Asian diabetic patients? Analysis from the Japan Diabetes Complications Study. Diabetes Care. 2005;28(6):1463-71.

10. Bruno G, Merletti F, Biggeri A, et al. Metabolic syndrome as a predictor of all-cause and cardiovascular mortality in type 2 diabetes: the Casale 
Monferrato Study. Diabetes Care. 2004;27(11): 2689-94.

11. Zinman B, Wanner C, Lachin JM, et al. Empagliflozin, cardiovascular outcomes, and mortality in type 2 diabetes. N Engl J Med. 2015;373(22): 2117-28.

12. Neal B, Perkovic V, Mahaffey KW, et al. Canagliflozin and cardiovascular and renal events in type 2 diabetes. N Engl J Med. 2017;377(7):644-57.

13. Wiviott SD, Raz I, Bonaca MP, et al. Dapagliflozin and cardiovascular outcomes in type 2 diabetes. N Engl J Med. 2019;380(4):347-57.

14. Marso SP, Daniels GH, Brown-Frandsen K, et al. Liraglutide and cardiovascular outcomes in type 2 diabetes. N Engl J Med. 2016;375(4):311-22.

15. Marso SP, Bain SC, Consoli A, et al. Semaglutide and cardiovascular outcomes in patients with type 2 diabetes. N Engl J Med. 2016;375(19):1834-44.

16. Nauck MA, Vilsboll T, Gallwitz B, Garber A, Madsbad $S$. Incretin-based therapies: viewpoints on the way to consensus. Diabetes Care. 2009;32[Suppl 2]:S223-31.

17. Kim YG, Hahn S, Oh TJ, Kwak SH, Park KS, Cho YM. Differences in the glucose-lowering efficacy of dipeptidyl peptidase-4 inhibitors between Asians and non-Asians: a systematic review and metaanalysis. Diabetologia. 2013;56(4):696-708.

18. Yamamoto-Honda R, Takahashi Y, Mori Y, et al. Changes in antidiabetic drug prescription and glycemic control trends in elderly patients with type 2 diabetes mellitus from 2005 to 2013: an analysis of the national center diabetes database (NCDD-03). Int Med. 2018;57(9):1229-40.

19. Mita T, Katakami N, Yoshii H, et al. Alogliptin, a dipeptidyl peptidase 4 inhibitor, prevents the progression of carotid atherosclerosis in patients with type 2 diabetes: the study of preventive effects of alogliptin on diabetic atherosclerosis (SPEAD-A). Diabetes Care. 2016;39(1):139-48.

20. Mita T, Katakami N, Shiraiwa T, et al. Sitagliptin attenuates the progression of carotid intima-media thickening in insulin-treated patients with type 2 diabetes: the Sitagliptin Preventive Study of IntimaMedia Thickness Evaluation (SPIKE): a randomized controlled trial. Diabetes Care. 2016;39(3):455-64.
21. McGill JB. The SGLT2 inhibitor empagliflozin for the treatment of type 2 diabetes mellitus: a bench to bedside review. Diabetes Ther. 2014;5(1):43-63.

22. Yamagishi $\mathrm{K}$, Iso $\mathrm{H}$. The criteria for metabolic syndrome and the national health screening and education system in Japan. Epidemiol Health. 2017;39:e2017003.

23. Giorgino F, Home PD, Tuomilehto J. Glucose control and vascular outcomes in type 2 diabetes: is the picture clear? Diabetes Care. 2016;39[Suppl 2]:S187-95.

24. Mishriky BM, Cummings DM, Tanenberg RJ. The efficacy and safety of DPP4 inhibitors compared to sulfonylureas as add-on therapy to metformin in patients with Type 2 diabetes: a systematic review and meta-analysis. Diabetes Res Clin Pract. 2015;109(2):378-88.

25. Ou HT, Chang KC, Li CY, Wu JS. Risks of cardiovascular diseases associated with dipeptidyl peptidase- 4 inhibitors and other antidiabetic drugs in patients with type 2 diabetes: a nation-wide longitudinal study. Cardiovasc Diabetol. 2016;15:41.

26. Matikainen N, Manttari S, Schweizer A, et al. Vildagliptin therapy reduces postprandial intestinal triglyceride-rich lipoprotein particles in patients with type 2 diabetes. Diabetologia. 2006;49(9): 2049-57.

27. Mulvihill EE, Drucker DJ. Pharmacology, physiology, and mechanisms of action of dipeptidyl peptidase-4 inhibitors. Endocr Rev. 2014;35(6):9921019.

28. Salim HM, Fukuda D, Higashikuni Y, et al. Teneligliptin, a dipeptidyl peptidase- 4 inhibitor, attenuated pro-inflammatory phenotype of perivascular adipose tissue and inhibited atherogenesis in normoglycemic apolipoprotein-E-deficient mice. Vascul Pharmacol. 2017;96-98:19-25.

29. Examination Committee of Criteria for "Obesity Disease" in Japan and Japan Society for the Study of Obesity. New criteria for obesity disease in Japan. Circ J. 2002;66(11):987-92.

30. WHO Expert Consultation. Appropriate body-mass index for Asian populations and its implications for policy and intervention strategies. Lancet. 2004;363(9403):157-63. 